\title{
Delayed Terminal Ileal Perforation in a Relapsed/Refractory B-Cell Lymphoma Patient with Rapid Remission Following Chimeric Antigen Receptor T-Cell Therapy
}

\author{
Yongxian $\mathrm{Hu}, \mathrm{MD}, \mathrm{PhD}$ \\ Jiasheng Wang, MD \\ Chengfei $\mathrm{Pu}, \mathrm{PhD}^{2}$ \\ Kui Zhao, MD, $\mathrm{PhD}^{3}$ \\ Qu Cui, MD, PhD \\ Guoqing Wei, MD, PhD \\ Wenjun Wu, MD, PhD' \\ Lei Xiao, $\mathrm{PhD}^{2}$ \\ Yang Xiao, $\mathrm{MD}, \mathrm{PhD}^{2}$ \\ Jinping Wang, $M D^{2}$ \\ Zhao Wu, $\mathrm{PhD}^{2}$ \\ He Huang, MD, $\mathrm{PhD}{ }^{1}$
}

${ }^{1}$ Bone Marrow Transplantation Center, The First Affiliated Hospital, Zhejiang

University School of Medicine, Hangzhou, 'Innovative Cellular Therapeutics Co., Ltd., Shanghai, ${ }^{3}$ PET/CT Center, The First Affiliated Hospital, Zhejiang University School of Medicine, Hangzhou, ${ }^{4}$ Department of Hematology, Beijing Tiantan Hospital, Capital Medical University, Beijing, China

Correspondence: He Huang, MD, PhD Bone Marrow Transplantation Center, The First Affiliated Hospital, Zhejiang University School of Medicine,

No. 79 Qingchun Road, Hangzhou, China

Tel, Fax: 86-0571-87236706

E-mail: huanghe@zju.edu.cn

Co-correspondence: Zhao Wu, PhD Innovative Cellular Therapeutics Co., Ltd., Room 201, Building 4, 998 Halei Road,

Shanghai, 201203, China

Tel: 86-21-58950719

Fax: 86-21-58950719

E-mail:wuzhao@sidansai.com

Received October 8, 2017

Accepted January 30, 2018

Published Online February 5, 2018

*Yongxian $\mathrm{Hu}$, Jiasheng Wang and Chengfei $\mathrm{Pu}$ contributed equally to this work.
Chimeric antigen receptor T-cell strategy targeting CD19 (CART19) has prominent anti-tumor effect for relapsed/refractory B-cell lymphomas. CART19-associated complications have been gradually recognized, however, late-onset complications have not been extensively studied. Herein, for the first time we report a diffuse large B-cell lymphoma patient with terminal ileum involvement obtained rapid remission and developed spontaneous terminal ileal perforation 38 days following CART19 infusion. The late-onset perforation reminds us that, for the safety of CART treatment, more cautions are warranted for the management of delayed Gl complications.

\section{Key words}

Refractory/relapse, B-cell lymphoma,

Chimeric antigen receptor T cells, Terminal ileal perforation 


\section{Introduction}

Adoptive T-cell therapy with genetically engineered chimeric antigen receptor (CAR) T-cell strategy targeting CD19 (CART19) has shown great potential for the treatment of B-cell malignancies, including acute lymphoblastic leukemia, chronic lymphocytic leukemia, and non-Hodgkin lymphoma. For relapsed/refractory B-cell lymphomas, CART19 therapy has demonstrated an overall response rate of $71 \%-73 \%$ and a complete remission (CR) rate of 50\%-57\% in recent trials [1-3]. Despite the promising response rates, safety issues still remain as a major concern for CART19 therapy. Cytokine release syndrome (CRS), neurological toxicities, and B-cell aplasia are most commonly encountered adverse events which have been widely recognized [4]. These adverse events occur hours or days following CART19 infusion and are usually well managed with aggressive supportive measures at early stage. On the contrary, lateonset complications of CAR T-cell therapy have not been extensively studied. Despite the scarcity of description, an early recognition of late-onset complications would be important to improve patients' long-term outcomes. And, the recognition of late-onset complications would shed light on CART19 biological behaviors in vivo. Herein, for the first time we report a case of diffuse large B-cell lymphoma (DLBCL) patient with terminal ileum involvement obtained rapid remission but developed spontaneous terminal ileal perforation 38 days following CART19 infusion.

\section{Case Report}

The 36-year-old male patient had a lengthy and complicated oncological history. He initially presented to a local hospital in 2003 due to an enlarged left tonsil. An excisional biopsy confirmed a diagnosis of DLBCL, and no other enlarged lymph nodes were appreciated at that time. Then, the patient initiated a six-cycle therapy of EPOCH (cyclophosphamide, doxorubicin, etoposide, vincristine, and prednisone) and achieved CR. A routine follow-up in June 2014 noted multiple nodal enlargements involving bilateral posterior cervical and axillary lymph nodes. Positron emission tomography/computed tomography (PET/CT) scan revealed intensive uptakes in the aforementioned sites, as well as the terminal ileum and the ascending colon. Then, a following CT-guided biopsy on the ascending colon mass was performed and afterwards confirmed a relapse of DLBCL (non-germinal center B cell type). After four cycles of R-CHOP (cyclophosphamide, doxorubicin, vincristine, and prednisone plus rituximab) treatment, a repeat PET/CT scan showed a partial remission with residual tumor activities in the terminal ileum. Two more cycles of R-IVAC (etoposide, ifosfamide, and cytarabine plus rituximab) were administered but still failed to achieve CR. The patient continued an alternative therapy and finally achieved CR (confirmed by PET/CT) after three more cycles of chemotherapy with rituximab, paclitaxel, and doxorubicin. Subsequently, the patient underwent autologous hematopoietic stem cell transplantation in March 2015 with conditioning regimen of carmustine, cytarabine, etoposide, and methotrexate. Seven months later, a repeat PET/CT in October 2015 revealed disseminated lymphoma relapsing regions including the terminal ileum, appendix, liver, mesentery lymph nodes, the left ilium, left femur, as well as axillary and cervical lymph nodes. The patient was treated by a following palliative therapy with thalidomide and rituximab until increased tumor burdens caused severe abdominal and back pain in May 2016. Given the patient's good performance status, a cycle of GDP (gemcitabine, dexamethasone, and cisplatin) was used before the primary disease progressed. Then, the patient was recruited to a CART19 clinical trial (Chictr.org N, ChiCTROCC-15007008) in December 2016 under informed consent. A pre-recruitment PET/CT showed increased activities in the terminal ileum, mesentery lymph nodes, the pancreatic tail, left femur and vertebral body of S2 (Fig. 1A). After a lymphocyte-depleting chemotherapy by FC regimen (fludarabine $30 \mathrm{mg} / \mathrm{m}^{2}$ day 1 to 3 , cyclophosphamide $500 \mathrm{mg} / \mathrm{m}^{2}$ day 2 to 3), CART19s were generated as previously reported $[5,6]$. The patient received an infusion of CART19s with a dose of $1.0 \times 10^{7}$ CART19s per kilogram.

After infusion, CART19s engrafted and expanded in peripheral blood. The patient developed fever (Fig. 1C) with marked elevation of serum interleukin (IL)-6, IL-10, interferon $\gamma($ IFN- $\gamma$ ), C-reactive protein, D-dimer, and ferritin 2 days after the infusion (Fig. 1D); grade 2 CRS was diagnosed. The serum cytokines fell into normal ranges within 4 days of supportive care. A follow-up PET/CT 30 days after the infusion showed a CR (Fig. 1B). However, 38 days after CART19 infusion, the patient suddenly developed severe abdominal pain with exacerbated diffuse tenderness and guarding. An exploratory laparotomy observed a perforation in the terminal ileum at the site coincident with the site of lymphoma involvement indicated in PET/CT scan before the CART19 infusion (Fig. 2A and B). The perforation was subsequently repaired and the pathology of resected ileum showed concomitant partial necrotic tissues without infiltration of lymphoma cells or CART19 (Fig. 2C and D). No elevation of IL-6, IL-10, INF- $\gamma$, granzyme B, or other inflammatory cytokines was noted. Detections of serum cytomegalo-virus-DNA and EBV-DNA during perforation showed negative results. Three months after surgery, the latest follow-up PET/CT 

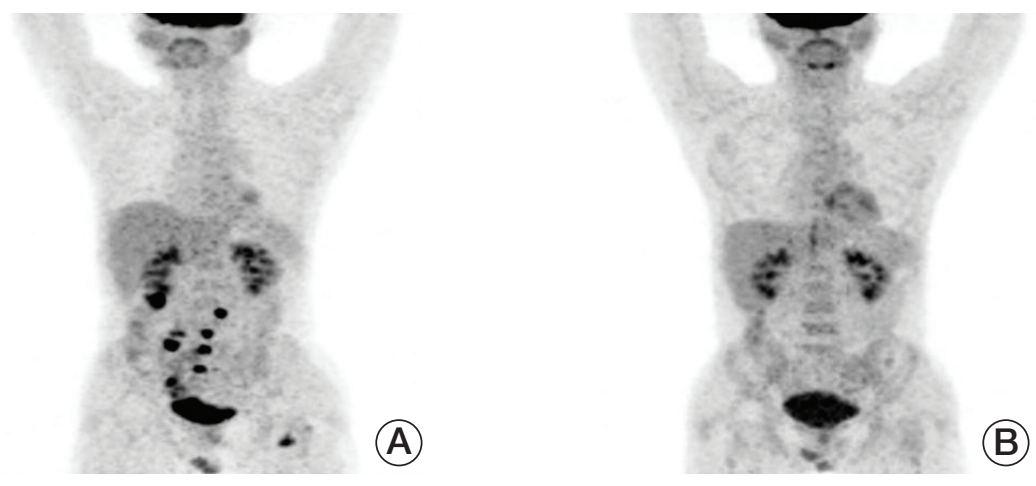

C
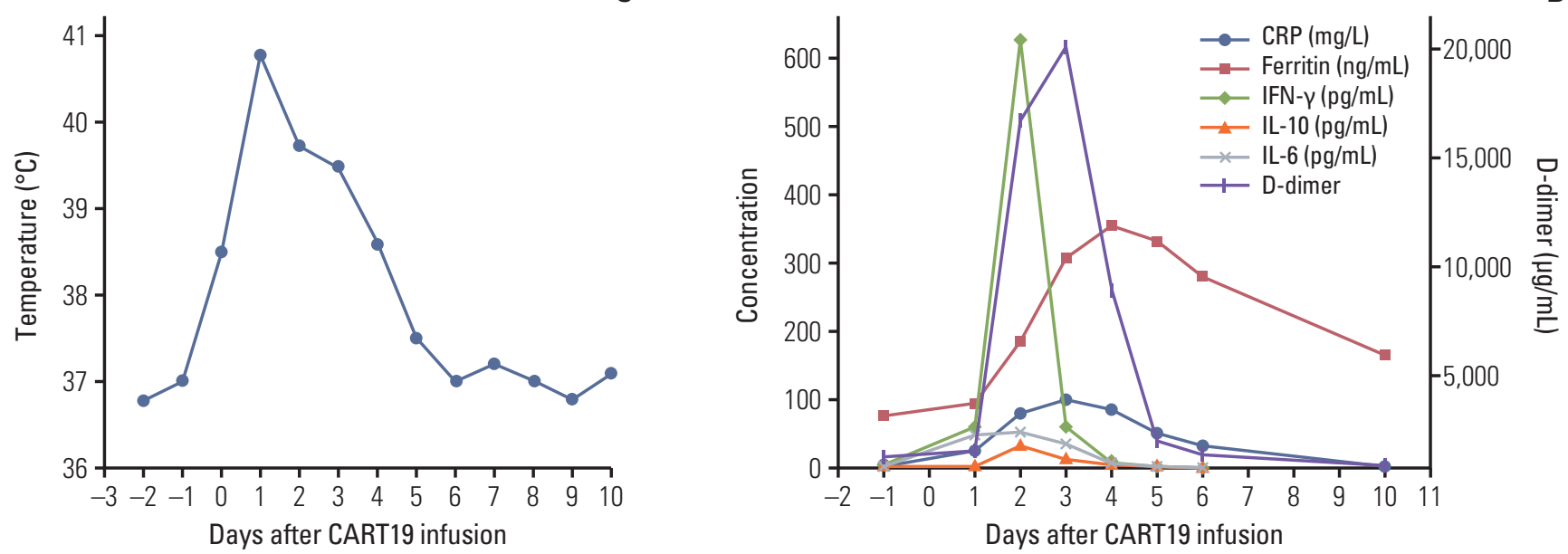

Fig. 1. The patient achieved complete remission 30 days after chimeric antigen receptor T-cell strategy targeting CD19 (CART19s) infusion with grade 2 cytokine release syndrome. (A) Positron emission tomography/computed tomography (PET/CT) showed lymphoma activities in the terminal ileum, the mesentery lymph nodes, the pancreatic tail, the left femur and the vertebral body of S2 pre-CART19 treatment. (B) PET/CT showed a complete remission 30 days post-CART19 treatment. (C) The patient developed fever 6 hours after CART19 infusion with the highest temperature $40.8^{\circ} \mathrm{C}$ and decreased to normal level after 5 days. (D) Serum levels of interleukin (IL)-6, IL-10, interferon $\gamma$ (IFN- $\gamma$ ), C-reactive protein (CRP), D-dimer, and ferritin were elevated 2 days after the infusion and fell into the normal range within 4 days with supportive care.

scan showed no observable residual lymphoma in ileal region adjacent to the perforated site.

\section{Discussion}

In this study, we reported a case of delayed terminal ileal perforation following rapid remission of CART19 therapy in a relapsed/refractory DLBCL patient. Such perforation case has not been reported previously. The patient achieved CR at 1 month after CART19s infusion, indicating that CART19s could rapidly migrate to lymphoma sites and eradicate lymphoma cells. Patients under chemotherapy usually achieve $\mathrm{CR}$ after four to six courses of treatment. The rapid remission by CART19 therapy potentiates a superior option for particular patients in the future.

Bowel perforation events have been reported in around 9\% of lymphoma patients with gastrointestinal (GI) involvement, of which 55\% occurred after chemotherapy [7]. In terms of histology, DLBCL was most commonly observed and associated with the highest frequency of perforation [8]. The small bowel was not the most commonly affected site, 

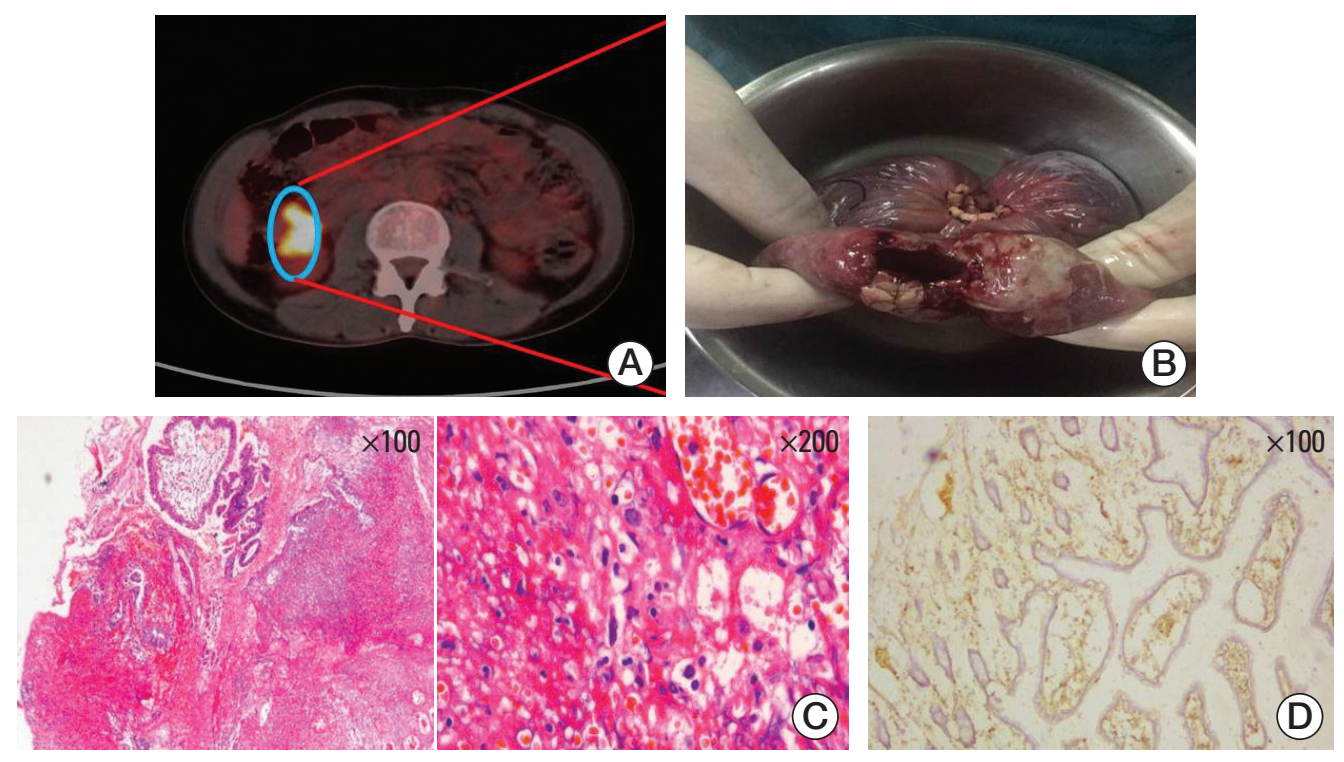

Fig. 2. (A, B) In the exploratory laparotomy, a perforation in the terminal ileum (B) was discovered at the site coincident with the location of lymphoma involvement reflected in positron emission tomography / computed tomography scan before chimeric antigen receptor T-cell strategy targeting CD19 (CART19) infusion (A). (C) Pathological findings by H\&E staining showed no lymphoma cells and partial necrotic tissue. (D) Immunochemical staining revealed a negative result for CART19.

however, was at the highest risk of perforation [8,9]. The timing of GI perforation after the initiation of chemotherapy varied from 4 days to more than 5 weeks in previous reports [10-12]. Vaidya et al. [7] described that about half of the perforation post chemotherapy occurred within the first month. The late on-set of perforation after chemotherapy turns to be related with tumor necrosis and local inflammation, since a large portion of patients with delayed perforation had no viable residual tumor tissues at the perforation site confirmed by pathology [7]. Notably, bowel perforation after chemotherapy is associated with a high mortality, an early recognition and a timely treatment are critical to improve the overall survival.

Our patient had a delayed terminal ileal perforation 38 days following CART19 infusion. The delayed presentation of symptoms posed a great challenge for timely diagnosis and treatment. As most CART19 complications occur hours or days after infusion, few late-onset complications have been reported in reported clinical trials. There have been reports of delayed CRS that occurred weeks after CAR T-cell infusion [13-15]. The late manifestation of CRS seemed to be related with late in vivo CAR T-cell expansion [11]. GI complications in lymphoma patients after CAR T-cell therapy were rare in clinical trials. Turtle et al. [2] described a patient with known GI invasive lymphoma mass died from a fatal GI hemorrhage after a high dose CAR T-cell infusion. Wang et al. [15] also described a patient with submucosal involve- ment of stomach lymphoma died from a massive GI hemorrhage 3 weeks after CART-20 infusion, whose late presentation of GI hemorrhage was correlated with delayed elevation of cytokines. No elevated cytokines or CART19s expansion were observed in our patient during the ileal perforation. Also the CAR T-cells were not identified from the ileal tissue. We hypothesize that tumor necrosis might contribute to the later perforation event. The high dose-intensity chemotherapy increases the risk of perforation in GI DLBCL patients, while patients undergoing CAR T-cell therapy are facing a higher risk of GI perforation, given the consistent and vigorous anti-tumor effects by CAR T-cells. Moreover, same as most late-onset GI perforations after chemotherapy, our patient stayed in CR during GI perforation, indicating that CART19 therapy and chemotherapy might share a similar mechanism in late-onset perforation. As we know, patients early after CART19 treatment might suffer from pancytopenia and abnormal coagulation function. When GI perforation occurred at this stage, there was almost no chance for surgery, which would lead to a high mortality for these patients. In this case, the ileal perforation occurred after 1 month of CART19 therapy, at a stage the patient spared early complications and achieved CR. The late onset of perforation reminds us that, for the safety of CART19 treatment, more cautions are still warranted to manage delayed GI complications in those patients.

Collectively, perforation of GI lymphomas after chemo- 
therapy has been well recognized while this case was the first report of perforation after CART19 treatment. The risk of GI perforation should always be aware of when treating a lymphoma patient with GI involvement. Additionally, this case brought forward another consideration that patients with GI lymphoma should be carefully evaluated before CART19 treatment. Along with the rapid evolvement of CAR T-cell therapy, an improved recognition of potential perforation complication is no-doubt critical for each involved patient. Still, more clinical trials are needed to address the location and timing of perforation in the disease course after CART19 therapy, to verify the probability and necessity of surgical intervention for these patients.

\section{Conflicts of Interest}

Conflict of interest relevant to this article was not reported.

\section{Acknowledgments}

This work was supported by grants from 973 Program (2015CB964900), the Natural Science Foundation of China (81230014, 81470341, 81500157, 81770201, 81730008), Key Project of Science and Technology Department of Zhejiang Province (2015C03G2010091).

\section{References}

1. Kochenderfer JN, Somerville RP, Lu T, Shi V, Bot A, Rossi J, et al. Lymphoma remissions caused by anti-CD19 chimeric antigen receptor $\mathrm{T}$ cells are associated with high serum interleukin-15 levels. J Clin Oncol. 2017;35:1803-13.

2. Turtle CJ, Hanafi LA, Berger C, Gooley TA, Cherian S, Hudecek M, et al. CD19 CAR-T cells of defined CD4+:CD8+ composition in adult B cell ALL patients. J Clin Invest. 2016;126: 2123-38.

3. Locke FL, Neelapu SS, Bartlett NL, Siddiqi T, Chavez JC, Hosing CM, et al. Phase 1 results of ZUMA-1: a multicenter study of KTE-C19 anti-CD19 CAR T cell therapy in refractory aggressive lymphoma. Mol Ther. 2017;25:285-95.

4. Brudno JN, Kochenderfer JN. Toxicities of chimeric antigen receptor T cells: recognition and management. Blood. 2016; 127:3321-30.

5. Hu Y, Sun J, Wu Z, Yu J, Cui Q, Pu C, et al. Predominant cerebral cytokine release syndrome in CD19-directed chimeric antigen receptor-modified T cell therapy. J Hematol Oncol. 2016;9:70.

6. Hu Y, Wu Z, Luo Y, Shi J, Yu J, Pu C, et al. Potent antileukemia activities of chimeric antigen receptor-modified $\mathrm{T}$ cells against CD19 in Chinese patients with relapsed/ refractory acute lymphocytic leukemia. Clin Cancer Res. 2017;23: 3297-306.

7. Vaidya R, Habermann TM, Donohue JH, Ristow KM, Maurer MJ, Macon WR, et al. Bowel perforation in intestinal lymphoma: incidence and clinical features. Ann Oncol. 2013;24: 2439-43.

8. Vaidya R, Witzig TE. Incidence of bowel perforation in gastrointestinal lymphomas by location and histology. Ann
Oncol. 2014;25:1249-50.

9. Ara C, Coban S, Kayaalp C, Yilmaz S, Kirimlioglu V. Spontaneous intestinal perforation due to non-Hodgkin's lymphoma: evaluation of eight cases. Dig Dis Sci. 2007;52:1752-6.

10. Wada M, Onda M, Tokunaga A, Kiyama T, Yoshiyuki T, Matsukura N, et al. Spontaneous gastrointestinal perforation in patients with lymphoma receiving chemotherapy and steroids: report of three cases. Nihon Ika Daigaku Zasshi. 1999; 66:37-40.

11. Zhai L, Zhao Y, Lin L, Tian Y, Chen X, Huang H, et al. NonHodgkin's lymphoma involving the ileocecal region: a singleinstitution analysis of 46 cases in a Chinese population. J Clin Gastroenterol. 2012;46:509-14.

12. Maisey N, Norman A, Prior Y, Cunningham D. Chemotherapy for primary gastric lymphoma: does in-patient observation prevent complications? Clin Oncol (R Coll Radiol). 2004;16: 48-52.

13. Porter DL, Hwang WT, Frey NV, Lacey SF, Shaw PA, Loren $\mathrm{AW}$, et al. Chimeric antigen receptor $\mathrm{T}$ cells persist and induce sustained remissions in relapsed refractory chronic lymphocytic leukemia. Sci Transl Med. 2015;7:303ra139.

14. Kebriaei P, Singh H, Huls MH, Figliola MJ, Bassett R, Olivares $\mathrm{S}$, et al. Phase I trials using Sleeping Beauty to generate CD19specific CAR T cells. J Clin Invest. 2016;126:3363-76.

15. Wang Y, Zhang WY, Han QW, Liu Y, Dai HR, Guo YL, et al. Effective response and delayed toxicities of refractory advanced diffuse large B-cell lymphoma treated by CD20-directed chimeric antigen receptor-modified T cells. Clin Immunol. 2014;155:160-75. 